\title{
Effect of combined 5-aza-2'deoxycytidine and cisplatin treatment on the P15 lung adenocarcinoma cell line
}

\author{
KAISHAN LIU, WENYAN HUANG, WEISONG GAO and WENFANG HE \\ Department of Pathology, School of Medicine, Jinan University, Guangzhou, Guangdong 510632, P.R. China
}

Received May 15, 2014; Accepted February 10, 2015

DOI: $10.3892 / 01.2015 .2986$

\begin{abstract}
Aberrant promoter hypermethylation resulting in the epigenetic silencing of apoptosis-associated genes is a key process in the chemotherapeutic treatment of cancer. The nucleoside analog, 5-aza-2'deoxycytidine (DAC), inhibits the activity of DNA methyltransferase enzymes and is able to restore the expression levels of genes that have been silenced by aberrant DNA methylation. The aim of the present study was to investigate the effect of combined treatment with DAC and cisplatin (CDDP) on the lung adenocarcinoma cell line, P15. Growth inhibition was examined using a clone formation assay and growth inhibitory activities by cell counting during treatment with DAC alone, CDDP alone or DAC followed by CDDP. In addition, changes in the mRNA expression levels of various apoptosis-associated genes following treatment with increasing concentrations of DAC were determined using reverse transcription-polymerase chain reaction. Furthermore, terminal deoxynucleotidyl transferase-mediated dUTP nick end labeling (TUNEL) analysis was used to detect the number of apoptotic P15 tumor cells following treatment with DAC and/or CDDP. The results indicated that DAC treatment alone restored the mRNA expression levels of $p 73, p 16^{I N K 4 a}$, B-cell lymphoma (Bcl)-2-associated agonist of cell death and Bcl-2-associated X protein. In addition, combined therapy with DAC and CDDP was found to significantly suppress the growth of P15 tumor cells compared with DAC or CDDP treatment alone. In conclusion, DAC may enhance the chemosensitivity of the P15 cell line to treatment with CDDP.
\end{abstract}

Correspondence to: Dr Kaishan Liu, Department of Pathology, School of Medicine, Jinan University, 601 West Huangpu Road, Guangzhou, Guangdong 510632, PR. China

E-mail: tlksh@jnu.edu.cn

Abbreviations: DAC, 5-aza-2'deoxycytidine; CDDP, cisplatin; NSCLC, non-small-cell lung cancer; RT-PCR, reverse transcription-polymerase chain reaction; TUNEL, terminal deoxynucleotidyl transferase-mediated dUTP nick end labeling

Key words: methylation, 5-aza-2'deoxycytidine, cisplatin, chemosensitivity, apoptosis

\section{Introduction}

Lung cancer is a leading cause of cancer-associated mortality worldwide and the most common type is non-small cell lung cancer (NSCLC) (1). Despite progress in the development of molecular-targeted therapeutic agents and surgical approaches, chemotherapy remains an important strategy for the treatment of NSCLC. A combination of a next-generation cytotoxic agents and a platinum compound is the standard treatment regimen for patients with advanced $\operatorname{NSCLC}(2,3)$. The cytotoxicity of cisplatin (CDDP), a platinum compound used in chemotherapy, is predominantly due to its interaction with DNA, forming DNA adducts that result in the activation of a number of apoptosis signaling pathways (4). However, the resistance of NSCLC cells to CDDP, which is currently a barrier in the treatment of patients with NSCLC, is associated with inactivation of these apoptosis signaling pathways $(5,6)$.

In tumor cells, numerous apoptosis-associated genes appear to have methylated $\mathrm{CpG}$ islands. DNA methylation is a crucial regulator in various biological processes; however, aberrant $\mathrm{CpG}$ island hypermethylation in gene promoter regions may impact the cell cycle, proliferation, apoptosis, metastasis, drug resistance and intracellular signaling. Therefore, the occurrence of this aberrant hypermethylation is important in carcinogenesis and cancer treatment. DNA hypermethylation is an epigenetic modification that leads to the transcriptional silencing of regulatory genes in various types of human cancer. Furthermore, DNA methyltransferase (DNMT) enzymes are critical for the maintenance of normal DNA methylation $(7,8)$.

The expression of apoptosis-associated genes, which is silenced by aberrant DNA hypermethylation, can be restored by the pyrimidine nucleoside analog of cytosine, 5-aza-2'deo xycytidine (DAC) $(9,10)$. The loss of DNA hypermethylation occurs through the formation of a covalent complex between DAC and DNMTs (11). Therefore, the clinical application of DAC in the treatment of patients with myelodysplastic syndrome (MDS) resulted in significant demethylation of apoptosis-associated genes in certain cases (12).

The aim of the present study was to evaluate the effect of combined therapy with DAC and CDDP on the human lung adenocarcinoma cell line, P15. In addition, the mRNA expression levels of six associated genes, including $p 73$, $\mathrm{B}$-cell lymphoma (Bcl)- $\mathrm{x}_{\mathrm{L}}$; Bcl-2-associated agonist of cell death $(B A D)$; Bcl-2-associated $\mathrm{X}$ protein $(B a x), p 14^{A R F}$ and p16 ${ }^{I N K 4 a}$, were analyzed. 
Table I. Genes and primer sequences.

\begin{tabular}{|c|c|c|c|c|}
\hline \multirow[b]{2}{*}{ Gene } & \multicolumn{2}{|c|}{ Nucleotide sequence, 5'-3' } & \multirow{2}{*}{$\begin{array}{l}\text { Annealing } \\
\text { temperature, }{ }^{\circ} \mathrm{C}\end{array}$} & \multirow{2}{*}{$\begin{array}{l}\text { Product } \\
\text { size, bp }\end{array}$} \\
\hline & Forward primer & Reverse primer & & \\
\hline$\beta$-actin & ССTTCCTGGGCATGGAGTCCT & AATCTCATCTTGTTTTCTGCG & 55 & 407 \\
\hline$p 73$ & ACTTCAACGAAGGACAGTCTGCT & AATTCCGTCCCCACCTGTG & 63 & 142 \\
\hline$B c l-x_{L}$ & CATGGCAGCAGTAAAGCAAG & GCATTGTTCCCATAGAGTTCC & 55 & 351 \\
\hline$B A D$ & TTTAAGAAGGGACTTCCTCGCC & GAGCTTCCCCTGCCCAAGTT & 60 & 119 \\
\hline Bax & ATCCAGGATCGAGCAGGGCG & ACTCGCTCAGCTTCTTGGTG & 58 & 94 \\
\hline$p 14^{A R F}$ & GAGTGAGGGTTTTCGTGG & GCCCATCATCATGACCTG & 55 & 153 \\
\hline$p 16^{I N K 4 a}$ & CAACGCACCGAATAGTTACGG & GCGCAGTTGGGCTCCG & 55 & 105 \\
\hline
\end{tabular}

Bcl, B-cell lymphoma (Bcl); Bax, Bcl-2-associated X protein; BAD, Bcl-2-associated agonist of cell death.

\section{Materials and methods}

Cell culture and DAC treatment. The P15 cell line, which is derived from lung adenocarcinoma, was obtained from the Center for Experimental Animals of Sun Yat-sen University (Guangzhou, China). P15 cells were maintained in RPMI -1640 medium (Corning, Inc., Corning, New York, NY, USA) supplemented with $10 \%$ fetal bovine serum in an atmosphere of $5 \% \mathrm{CO}_{2}$ at $37^{\circ} \mathrm{C}$. The $\mathrm{P} 15$ cell culture was exposed to different concentrations of the demethylation agent, DAC (2, 5 and $10 \mu \mathrm{mol} / 1$; Sigma-Aldrich, St. Louis, MO, USA), for $72 \mathrm{~h}$ to assess growth inhibition and monitor the mRNA expression levels of specific genes.

Clone formation assay. The exponentially growing cells were trypsinized (Sigma-Aldrich) into a single cell suspension following treatment with $5 \mu \mathrm{M}$ DAC for $72 \mathrm{~h}$. Next, the cells were inoculated into 6-well plates, with each well containing 500 cells. The cells were treated with $0.05 \mu \mathrm{M}$ CDDP for $72 \mathrm{~h}$ and cultured at $37^{\circ} \mathrm{C}$ in an atmosphere of $5 \% \mathrm{CO}_{2}$ to facilitate colony formation for 12 days. After rinsing twice with phosphate-buffered saline (PBS), the colonies were fixed with $1 \mathrm{ml}$ methanol for $30 \mathrm{~min}$. The fixing solution was discarded and $1 \mathrm{ml}$ Giemsa dye (Wuhan Huamei Biotechnology Co., Ltd., Beijing, China) was applied for $30 \mathrm{~min}$ to stain the cells. Colonies containing a minimum of 50 viable cells were counted using an inverted fluorescence microscope (Olympus BX-51; Olympus Corporation, Tokyo, Japan) to quantify the survival fractions. The survival fraction was calculated using the following formula: Survival fraction $=$ number of colonies $/ 500$ cells .

Growth inhibitory activities of DAC. Cells $\left(0.25 \times 10^{4}\right)$ were seeded in triplicate in 24 -well plates two days prior to treatment (day -2). The seeded cells were divided into four groups as follows: untreated control; DAC alone; CDDP alone; and DAC followed by CDDP treatment groups. New medium containing $5 \mu \mathrm{M}$ of DAC (or PBS) was added to the appropriate groups on day 0 . On day 3 , the cells were rinsed with PBS and new medium containing $0.05 \mu \mathrm{M}$ CDDP was added to the CDDP alone and DAC followed by CDDP treatment groups, according to the manufacturer's instructions. The cells were digested with $0.25 \%$ trypsin and $0.5 \mathrm{M}$ EDTA and counted daily from seeding until day 6 .

Total RNA extraction and reverse transcription-polymerase chain reaction $(R T-P C R)$. Total RNA was extracted from the cells using TRIzol reagent (Omega Bio-Tek, Inc., Norcross, GA, USA), purified and dissolved in diethylpyrocarbonate-treated distilled water. Next, $1.0 \mu \mathrm{g}$ total RNA was reverse-transcribed using avian myeloblastosis virus reverse transcriptase (Takara Biotechnology Co., Ltd., Dalian, China). cDNA was then obtained and PCR amplification was performed using the primers indicated in Table I, under the following conditions: Initial denaturation at $94^{\circ} \mathrm{C}$ for $2 \mathrm{~min}$, followed by $30-35$ cycles of denaturation at $94^{\circ} \mathrm{C}$ for $30 \mathrm{sec}$, annealing at $55-63^{\circ} \mathrm{C}$ for $30 \mathrm{sec}$ and extension at $72^{\circ} \mathrm{C}$ for $1 \mathrm{~min}$. The PCR mixture consisted of: distilled $\mathrm{H}_{2} \mathrm{O} 12.5 \mu 1$, 5X PCR buffer $2 \mu \mathrm{l}$ (Takara Biotechnology Co., Ltd.), Taq enzyme $0.5 \mu 1$ (Takara Biotechnology Co., Ltd.), NTPs $2 \mu 1$ (Takara Biotechnology Co., Ltd.), forward primer $1 \mu \mathrm{l}$,reverse primer $1 \mu \mathrm{l}$,cDNA $1 \mu \mathrm{l}$. The primers were designed and synthesized by Takara Biotechnology Co. Ltd. The PCR products were electrophoresed on 3\% agarose gels, stained with ethidium bromide (Takara Biotechnology Co., Ltd.) and visualized under ultraviolet illumination using a Uvidoc gel documentation system (GelDoc XR, Bio-Rad Laboratories, Inc., Hercules, CA, USA). $\beta$-actin was selected as the endogenous reference gene. The six apoptosis-related genes, such as $p 73, B c l-x_{L}, B A D, B a x, p l 4^{A R F}$, and $p l 6^{I N K 4 a}$, were investigated by RT-PCR.

Terminal deoxynucleotidyl transferase (TdT)-mediated dUTP nick end labeling (TUNEL) analysis. TUNEL (Roche Diagnostics, Basel, Switzerland) was used to evaluate the treatment-associated apoptosis, according to the manufacturer's instructions. Cells $\left(0.25 \times 10^{4} /\right.$ well $)$ were seeded on the slides in 6-well plates and exposed to $5 \mu \mathrm{M}$ DAC for $72 \mathrm{~h}$, followed by $1.25 \mu \mathrm{M}$ CDDP for $72 \mathrm{~h}$. The slides were washed with PBS, fixed for 60 min with $4 \%$ paraformaldehyde and incubated with TUNEL detection solution containing TdT and fluorescein isothiocyanate (FITC)-labeled solution. DNA strand breaks were labeled with FITC-conjugated dUTP and the nuclei were visualized using an inverted fluorescence microscope (Olympus BX-51; Olympus Corporation). 
A
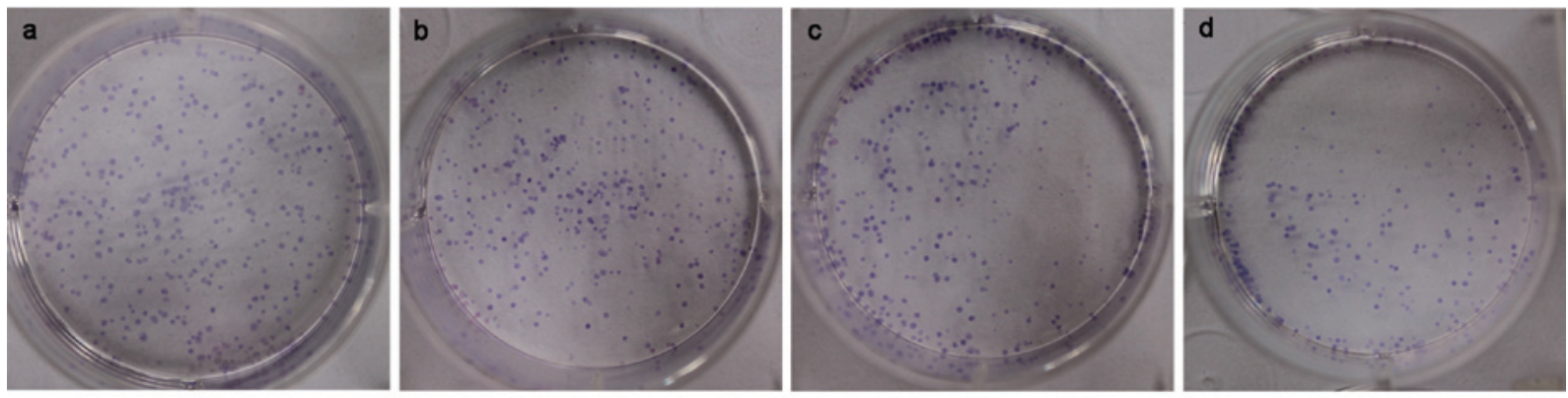

B

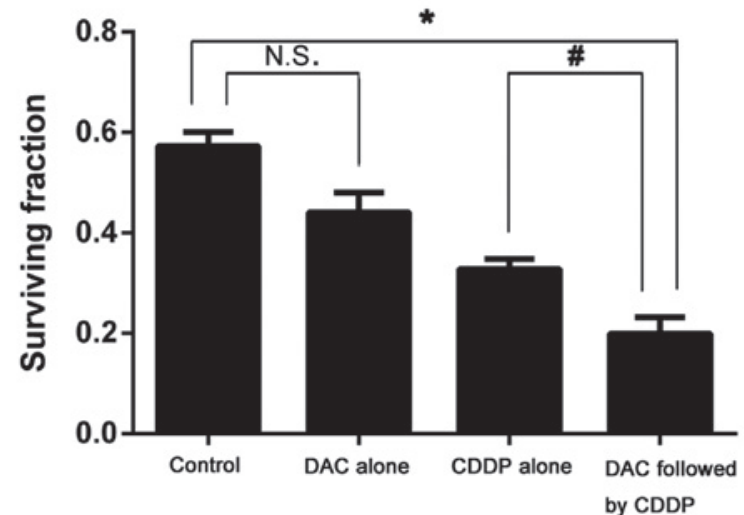

Figure 1. (A) Survival of (a) control cells; and cells treated with (b) DAC alone; (c) CDDP alone; and (d) DAC followed by CDDP, all stained with Giemsa dye. A higher number of stained cells indicates greater survival. Following DAC treatment for $72 \mathrm{~h}$, the P15 cells were exposed to the chemotherapeutic agent, CDDP, for $72 \mathrm{~h}$ and cultured for 12 days. (B) The extent of colony formation was quantified by clonogenic cell survival analysis. ${ }^{*} \mathrm{P}=0.001$ and ${ }^{\#} \mathrm{P}=0.029$ (Welch's t-test). N.S., no significant difference; DAC, 5-aza-2'deoxycytidine; CDDP, cisplatin.

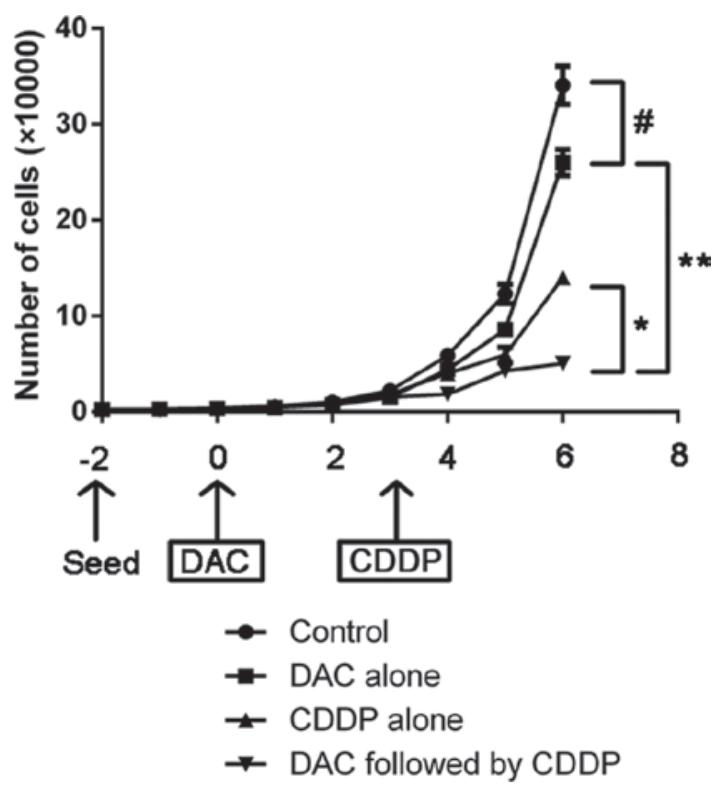

Figure 2. Growth curve of P15 cell proliferation following treatment with DAC and/or CDDP. Cells were divided into four groups (untreated control; DAC alone; CDDP alone; and DAC followed by CDDP) and the number of cells was counted daily following seeding until day $6 .{ }^{\prime \prime} \mathrm{P}=0.029,{ }^{*} \mathrm{P}<0.001$ and ${ }^{* *} \mathrm{P}=0.004$ were determined using Welch's t-test. DAC, 5-aza-2'deoxycytidine; CDDP, cisplatin.

Statistical analysis. Statistical analyses were performed using the SPSS software (version 16.0; SPSS Inc., Chicago, IL, USA). Survival and apoptosis were analyzed using Welch's $\mathrm{t}$-test and $\mathrm{P}<0.05$ was considered to indicate a statistically

\begin{tabular}{llllll} 
DAC & 0 & 2 & 5 & 10 & $(\mu \mathrm{M}) 72 \mathrm{~h}$ \\
\hline
\end{tabular}

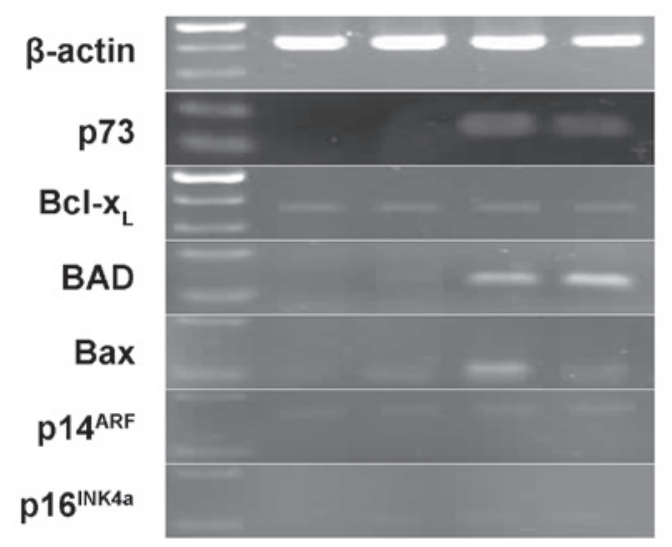

Figure 3. Following exposure to $0,2,5$ and $10 \mu \mathrm{M}$ DAC for $72 \mathrm{~h}$, the mRNA expression levels of six genes was detected by performing reverse transcription-polymerase chain reaction. DAC, 5-aza-2'deoxycytidine; Bcl, B-cell lymphoma (Bcl); Bax, Bcl-2-associated X protein; BAD, Bcl-2-associated agonist of cell death.

significant difference. Data are presented as the mean \pm standard deviation.

\section{Results}

Colony formation. The survival fraction for colony formation in cells treated with DAC alone $(0.440 \pm 0.009)$ was not significantly 

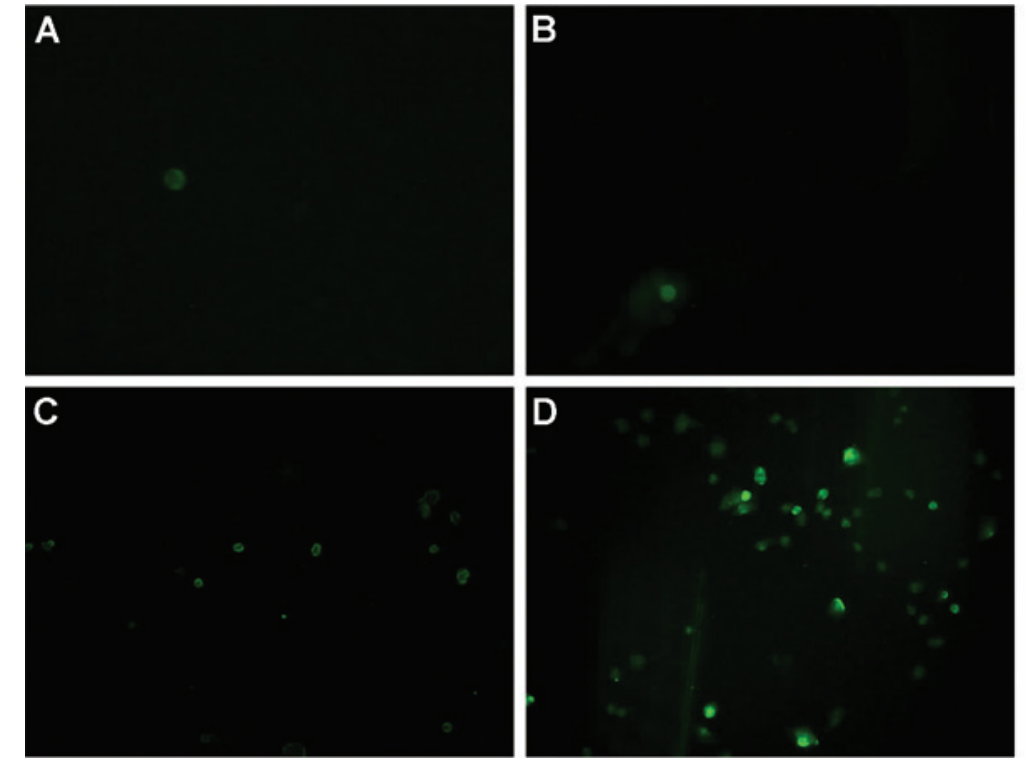

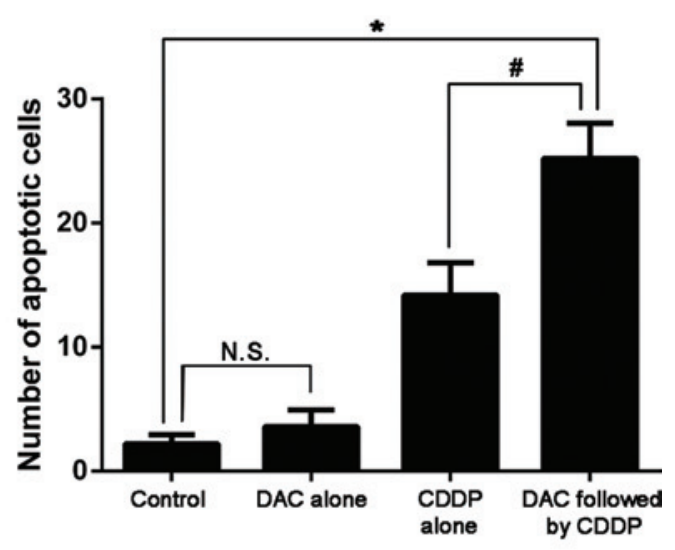

Figure 4. Apoptotic cells were detected by green fluorescence. Left panel: (A) Control; (B) DAC alone; (C) CDDP alone; and (D) DAC followed by CDDP groups. Right panel: The mean number of terminal deoxynucleotidyl transferase-mediated dUTP nick end labeling-positive cells is indicated in the bar graph. Error bars indicate the standard deviation form the mean. ${ }^{*} \mathrm{P}=0.001$ and ${ }^{\#} \mathrm{P}=0.022$ were determined using Welch's t-test. DAC, 5-aza-2'deoxycytidine; CDDP, cisplatin; N.S., no significant difference.

different from that of the control group $(0.574 \pm 0.004 ; \mathrm{P}>0.05)$. However, following combined treatment with DAC and CDDP, the survival fraction for colony formation was significantly decreased when compared with the DAC alone group (0.200 \pm 0.007 vs. $0.328 \pm 0.007$; $\mathrm{P}<0.05$; Fig. 1$)$.

Cell proliferation inhibition. The growth curve of cell proliferation following treatment with DAC and/or CDDP is indicated in Fig. 2. On day 6, the number of cells in the combined treatment group was $15 \%$ lower compared with the number of cells in the control group $(\mathrm{P}=0.004)$. Furthermore, cell proliferation was significantly more suppressed in DAC alone group compared with the control group, as well as in the combined DAC and CDDP treatment group compared with the CDDP alone group $(\mathrm{P}<0.05)$.

Effect of DAC on mRNA expression levels. Following exposure to different concentrations of DAC, the mRNA expression levels of six genes in the P15 cells was determined (Fig. 3). The P15 cells expressed no $p 73$ mRNA following treatment with 0 and $2 \mu \mathrm{M}$ DAC; however, equal levels of $p 73$ expression occurred upon treatment with 5 and $10 \mu \mathrm{M}$ DAC. Therefore, DAC appears to restore the expression of $p 73$ transcripts. In addition, no mRNA expression of $B A D$ was identified in the untreated P15 cells. By contrast, treatment with 5 and $10 \mu \mathrm{M}$ DAC markedly enhanced $B A D$ expression and treatment with $2 \mu \mathrm{M}$ DAC weakly enhanced $B A D$ expression. In addition, $p 16^{I N K 4 a}$ was expressed weakly upon treatment with 2, 5 and $10 \mu \mathrm{M}$ DAC, while Bax mRNA expression was evident at $5 \mu \mathrm{M}$ DAC, and weakly detected at 2 and $10 \mu \mathrm{M}$ DAC. However, the mRNA expression levels of $p 14^{A R F}$ and $B c l-x_{L}$ did not appear to be affected by DAC treatment.

Cell apoptosis. Apoptotic cells are illustrated by fluorescence in Fig. 4. A small number of apoptotic cells were identified in the untreated cells or the cells treated with DAC alone, with no statistically significant difference in the mean number of apoptotic cells observed between these two groups. The highest mean number of apoptotic cells was observed in the DAC and CDDP combination therapy group ( $\mathrm{P}=0.001$, vs. control group).

\section{Discussion}

Previous studies have indicated that methylation of specific genes resulting in epigenetic silencing occurs in lung cancer. These genes are involved in a number of processes, including cell cycle control, DNA repair, the regulation of cell differentiation and proliferation, and pro-apoptosis $(13,14)$. Therefore, the DNA methylation status of these genes may be used as a molecular marker for early diagnosis, prognosis, disease recurrence risk assessment or response to therapy in patients with lung cancer $(15,16)$.

The clinical efficacy of DAC therapy has yet to be fully investigated in solid tumors; however, the combination of DAC and a chemotherapeutic agent has been proven to exhibit a synergistic effect. For instance, Charlet et al (17) reported that the treatment of neuroblastoma cells with a combination of chemotherapeutic agents and DAC significantly increased the levels of apoptosis induced by CDDP, doxorubicin and etoposide therapy, when compared with treatment with these chemotherapeutic agents alone. Furthermore, Shang et al (18) demonstrated that combination therapy with DAC and CDDP may be a novel strategy to improve the clinical response rate of transitional cell carcinoma of the bladder.

p73 is a member of the p53 family and, therefore, p73 and p53 share significant homology in their DNA-binding domains. Similar to p53, p73 can elicit cancer cell apoptosis in response to DNA damage caused by CDDP-based chemotherapy. However, the $p 73$ gene is rarely mutated $(19,20)$. Alternatively, epigenetic 
silencing by promoter hypermethylation and complex formation with inhibitory proteins are two distinct inactivation mechanisms of $p 73$ (21). Epigenetic silencing of the $p 73$ gene via hypermethylation of its promoter was previously identified in MDS and non-Hodgkin lymphoma $(22,23)$. Furthermore, the loss of $p 73$ expression in six NSCLC cell lines was determined to be associated with $5^{\prime} \mathrm{CpG}$ island hypermethylation. In the $\mathrm{C} 57$ cell line, DAC was able to restore the mRNA expression of $p 73(24,25)$. Similarly, the present study identified that DAC was successful in restoring $p 73$ mRNA expression in the human lung adenocarcinoma cell line, P15 (Fig. 3). Furthermore, chemotherapeutic agent (CDDP)-induced cell death was increased in the $\mathrm{P} 15$ cell line when administered in combination with DAC (Fig. 4). Thus, p73 appeared to enhance the chemosensitivity of P15 tumor cells to CDDP, indicating a potential role of $p 73$ as a tumor suppressor in NSCLC.

p16 ${ }^{\mathrm{INK} 4 \mathrm{a}}$, which is known to participate in the regulation of the cell cycle, is frequently inactivated in a variety of human cancer types. Kim et al (26) proposed that abnormal methylation of $p 16^{I N K 4 a}$ was an early event of lung carcinogenesis. Furthermore, hypermethylation of $p 16^{I N K 4 a}$ was identified in plasma and sputum samples of patients with early lung cancer, as well as in lung cancer tissue samples $(27,28)$. These studies indicated that $p 16^{I N K 4 a}$ gene hypermethylation presented high specificity as the hypermethylation did not occur in healthy lung tissue. In addition, the abnormal methylation of $p 16^{I N K 4 a}$ is associated with poor prognosis in NSCLC patients (29) as the disruption of p16 ${ }^{I N K 4 a}$ cell cycle control would allow clonal expansion to occur. In the present study, clone formation and cell growth inhibitory assays demonstrated that the tumor cell growth was significantly inhibited upon treatment with DAC; this may indicate an association between restored expression of p16 ${ }^{I N K 4 a}$ and cell growth inhibition (Figs. 1, 2 and 3).

$B A D$ and Bax are pro-apoptotic genes of the Bcl-2 family; therefore, aberrant promoter methylation in these genes may affect the tumor cell apoptosis pathway $(30,31)$. In the present study, the treatment of P15 cells with DAC increased $B A D$ and Bax mRNA expression levels (Fig. 3). Although the mechanisms involved have yet to be fully elucidated, DAC may enhance pro-apoptosis in P15 cells through the restoration of $B A D$ and Bax genes (Fig. 4).

In conclusion, the present study demonstrated that the aberrant hypermethylation of various gene promoters affected the chemosensitivity of human lung adenocarcinoma cells to treatment with CDDP. Treatment of P15 cells with DAC restored the mRNA expression levels of $p 73, p 16^{I N K 4 a}, B A D$ and Bax. Furthermore, combined therapy with DAC and CDDP significantly suppressed the growth of lung tumor cells compared with DAC or CDDP treatment alone, indicating the potential of DAC in the treatment of NSCLC.

\section{Acknowledgements}

Data published in the present study were extracted from the medical thesis of Mr. Wenyan Huang.

\section{References}

1. Field JK, Oudkerk M, Pedersen JH and Duffy SW: Prospects for population screening and diagnosis of lung cancer. Lancet 382: 732-741, 2013
2. Reck M, Heigener DF, Mok T, Soria JC and Rabe KF: Management of non-small-cell lung cancer: recent developments. Lancet 382: 709-719, 2013.

3. Ozawa Y, Inui N, Naitoh T, et al: Phase II study of combination chemotherapy with S-1 and weekly cisplatin in patients with previously untreated advanced non-small cell lung cancer. Lung Cancer 63: 68-71, 2009.

4. Barr MP, Gray SG, Hoffmann AC, et al: Generation and characterisation of cisplatin-resistant non-small cell lung cancer cell lines displaying a stem-like signature. PloS One 8: e54193, 2013.

5. Sève $P$ and Dumontet $C$ : Chemoresistance in non-small cell lung cancer. Curr Med Chem Anticancer Agents 5: 73-88, 2005.

6. Almeida GM, Duarte TL, Farmer PB, Steward WP and Jones GD: Multiple end-point analysis reveals cisplatin damage tolerance to be a chemoresistance mechanism in a NSCLC model: implications for predictive testing. Int J Cancer 122: 1810-1819, 2008.

7. McCabe MT, Brandes JC and Vertino PM: Cancer DNA methylation: molecular mechanisms and clinical implications. Clin Cancer Res 15: 3927-3937, 2009.

8. Cheung HH, Lee TL, Rennert OM and Chan WY: DNA methylation of cancer genome. Birth Defects Res C Embryo Today 87: 335-350, 2009.

9. Fang JY, Yang L, Zhu HY, Chen YX, Lu J, Lu R, Cheng ZH, and Xiao SD: 5-Aza-2'-deoxycitydine induces demethylation and up-regulates transcription of p16INK4a gene in human gastric cancer cell lines. Chin Med J (Engl) 117: 99-103, 2004.

10. Chu LC, Eberhart CG, Grossman SA and Herman JG: Epigenetic silencing of multiple genes in primary CNS lymphoma. Int J Cancer 119: 2487-2491, 2006.

11. Karahoca M and Momparler RL: Pharmacokinetic and pharmacodynamic analysis of 5-aza-2'-deoxycytidine (decitabine) in the design of its dose-schedule for cancer therapy. Clin Epigenetics 5: 3, 2013

12. Mund C, Hackanson B, Stresemann C, Lübbert M and Lyko F: Characterization of DNA demethylation effects induced by 5 -aza-2'-deoxycytidine in patients with myelodysplastic syndrome. Cancer Res 65: 7086-7090, 2005.

13. Belinsky SA: Gene-promoter hypermethylation as a biomarker in lung cancer. Nat Rev Cancer 4: 707-717, 2004.

14. Belinsky SA: Silencing of genes by promoter hypermethylation: key event in rodent and human lung cancer. Carcinogenesis 26: 1481-1487, 2005.

15. Pfeifer GP and Rauch TA: DNA methylation patterns in lung carcinomas. Semin Cancer Biol 19: 181-187, 2009.

16. Fleischhacker M, Dietrich D, Liebenberg V, Field JK and Schmidt B: The role of DNA methylation as biomarkers in the clinical management of lung cancer. Expert Rev Respi Med 7: 363-383, 2013.

17. Charlet J, Schnekenburger M, Brown KW and Diederich M: DNA demethylation increases sensitivity of neuroblastoma cells to chemotherapeutic drugs. Biochem Pharmacol 83: 858-865, 2012.

18. Shang D, Liu Y, Matsui Y, et al: Demethylating agent 5-aza-2'-deoxycytidine enhances susceptibility of bladder transitional cell carcinoma to cisplatin. Urology 71: 1220-1225, 2008.

19. Han S, Sermba S, Abe T, Makino N, Furukawa T, Fukushige S, Takahashi H, Sakurada A, Sato M, Shiiba K, et al: Infrequent somatic mutations of the 773 gene in various human cancers. Eur J Surg Oncol 25: 194-198, 1999.

20. Yoshikawa H, Nagashima M, Khan MA, McMenamin MG Hagiwara K and Harris CC: Mutational analysis of p73 and p53 in human cancer cell lines. Oncogene 18: 3415-3421, 1999.

21. Maas AM, Bretz AC, Mack E and Stiewe T: Targeting p73 in cancer. Cancer Lett 332: 229-236, 2013.

22. Zhao Y, Fei C, Zhang X, et al: Methylation of the p73 gene in patients with myelodysplastic syndromes: correlations with apoptosis and prognosis. Tumour Biol 34: 165-172, 2013.

23. Pei JH, Luo SQ, Zhong Y, Chen JH, Xiao HW and Hu WX: The association between non-Hodgkin lymphoma and methylation of p73. Tumour Biol 32: 1133-1138, 2011.

24. Liu K, Zhan M and Zheng P: Loss of p73 expression in six non-small cell lung cancer cell lines is associated with $5^{\prime} \mathrm{CpG}$ island methylation. Exp Mol Pathol 84: 59-63, 2008.

25. Liu K, Zhuang X and Mai Z: p73 expression is associated with cellular chemosensitivity in human non-small cell lung cancer cell lines. Oncol Lett 5: 583-587, 2013.

26. Kim DH, Nelson HH, Wiencke JK, et al: p16(INK4a) and histology-specific methylation of $\mathrm{CpG}$ islands by exposure to tobacco smoke in non-small cell lung cancer. Cancer Res 61: 3419-3424, 2001 
27. Bearzatto A, Conte D, Frattini M, et al: p16(INK4A) hypermethylation detected by fluorescent methylation-specific PCR in plasmas from non-small cell lung cancer. Clin Cancer Res 8: 3782-3787, 2002.

28. Shin KC, Lee KH, Lee CH, Shin IH, Suh HS and Jeon CH: MAGE A1-A6 RT-PCR and MAGE A3 and p16 methylation analysis in induced sputum from patients with lung cancer and non-malignant lung diseases. Oncol Rep 27: 911-916, 2012.

29. Lou-Qian Z, Rong Y, Ming L, Xin Y, Feng J and Lin X: The prognostic value of epigenetic silencing of p16 gene in NSCLC patients: a systematic review and meta-analysis. PloS One 8: e54970, 2013.
30. Zinkel S, Gross A and Yang E: BCL2 family in DNA damage and cell cycle control. Cell Death Differ 13: 1351-1359, 2006.

31. Hervouet E, Vallette FM and Cartron PF: Impact of the DNA methyltransferases expression on the methylation status of apoptosis-associated genes in glioblastoma multiforme. Cell Death Dis 1: e8, 2010. 